\title{
The influence of the Great East Japan Earthquake on tuberculosis control in Japan
}

\author{
Akira Shimouchi, ab Noriko Kobayashi, ${ }^{b}$ Yoko Nagata, ${ }^{b}$ Minako Urakawa ${ }^{b}$ and Nobutatsu Ishkawa ${ }^{b}$ \\ Correspondence to Akira Shimouchi (email: ak-shimouchi@city.osaka.lg.jp).
}

$\mathrm{T}$ he Great East Japan Earthquake and subsequent tsunami hit the Pacific Ocean side of north-eastern Japan on 11 March $2011,{ }^{1}$ resulting in more than 18000 deaths and missing people in three prefectures: Iwate, Miyagi and Fukushima. $^{2}$ Of those deaths, $65 \%$ were aged 60 years and older, and more than $90 \%$ were caused by drowning. ${ }^{3}$ The earthquake also destroyed nuclear power plants in Fukushima, causing high levels of radioactive contamination. ${ }^{4}$ As a result, there were 386739 evacuees staying in 2182 temporary shelters such as community centres, schools and gymnasiums one week after the disaster. ${ }^{5}$

In Japan, tuberculosis (TB) control activities are conducted by public health centres (PHCs) and treatment support is provided by public health nurses (PHNs). This study describes the TB situation in the affected areas and assesses the effectiveness of Japan's TB control efforts after the disaster.

\section{METHODS}

We obtained data on casualties of the disaster from the National Police Agency and Ministry of Internal Affairs and Communications. ${ }^{2}$ From April 2011 to March 2014, teams of medical doctors and PHNs of the Japan Anti-Tuberculosis Association (JATA) visited eight PHCs and three hospitals for TB patient followup in the eight disaster-affected PHC areas where the mortality or missing rate was higher than $0.1 \%$. Data for each TB patient, including bacteriological test results, regimen and treatment outcome were collected by the PHNs for analysis. Information on individual TB patient support and TB outbreaks in shelters were collected at consultation meetings with local staff during the JATA team visits. ${ }^{6}$ TB outbreaks were confirmed by the interferon- $\gamma$ release assay as reported elsewhere. ${ }^{7,8}$

TB notification data at PHCs were obtained with permission of local governments. TB notification rates were compared between disaster-affected and nonaffected areas using the chi-square test. Analysis was conducted using Microsoft Excel (Microsoft Excel 2010, Redmond, USA). A $P$-value $<0.05$ was considered statistically significant. Ethical approval was obtained from the Research Institute of Tuberculosis, JATA.

\section{RESULTS}

There were 96 TB patients on treatment in the eight $\mathrm{PHC}$ areas at the time of the disaster. The consultation meetings revealed that no TB patients had defaulted from treatment in these areas.

\section{Death of TB patients from disaster}

Seven TB patients died during the disaster (five from PHC $\mathrm{D}$, one from $\mathrm{PHC} \mathrm{G}$ and one from $\mathrm{PHC} H$ ). Mortality of TB patients $(7.3 \%)$ was higher than that of the general population (1.3\%) in these areas. In the PHC D area, mortality of TB patients was much higher than that of the general population (23.8\% versus $2.7 \%$ ) (Table 1 ). Mortality of TB patients aged 60 years or older $(30.7 \%$, $4 / 13$ ) was higher than that of those younger than age 60 $(12.5 \%, 1 / 8)$ in this area.

\section{TB outbreak in shelters}

Two TB outbreaks in different shelters were reported in the disaster-affected PHC areas in 2011. The first 
Table 1. PHC areas with mortality or missing rates higher than $0.1 \%$ in three affected prefectures in Japan after the Great East Japan Earthquake, 2011

\begin{tabular}{|c|c|c|c|}
\hline PHC area & $\begin{array}{c}\text { Number of TB } \\
\text { patients on treatment }\end{array}$ & $\begin{array}{l}\text { Number of TB patients } \\
\text { dead or missing (\%) }\end{array}$ & $\begin{array}{l}\text { Death or missing rate of } \\
\text { general population }(\%)^{2}\end{array}$ \\
\hline A (Iwate) & 1 & $0 \quad(0)$ & 3.5 \\
\hline B (Iwate) & 6 & $0 \quad(0)$ & 4.2 \\
\hline C (Iwate) & 5 & $0 \quad(0)$ & 1.4 \\
\hline D (Miyagi) & 21 & $5 \quad(23.8)$ & 2.7 \\
\hline E (Miyagi) & 8 & $0 \quad(0)$ & 2.4 \\
\hline F (Miyagi) & 18 & $0 \quad(0)$ & 0.7 \\
\hline G (Fukushima) & 10 & $1 \quad(10.0)$ & 0.8 \\
\hline H (Fukushima) & 27 & 1 (3.7) & 0.1 \\
\hline Total & 96 & $7 \quad(7.3)$ & 1.3 \\
\hline
\end{tabular}

PHC, public health centre; and TB, tuberculosis.

outbreak involved an 80-year-old female staying in a 60 square-metre shelter with about 50 people. The ventilation was poor as windows were closed due to cold weather. Nine people were confirmed to have latent TB infection (LTBI). Another outbreak involved a 50-year-old male staying in a large shelter with about 2500 people. In the subdivision where the subject stayed, ventilation was poor due to low ceilings and the surrounding three walls. In this outbreak, two TB patients and 18 people with LTBI were identified.

\section{TB notification trend}

From 2010 to 2013, the annual TB notification rate did not change significantly in the eight disasteraffected PHC areas $(11.4,9.4,11.2$ and 9.9 per 100000 individuals, $P=0.262$ ) and in other $\mathrm{PHC}$ areas (12.0, 10.5, 10.3 and 11.1 per 100000 individuals, $P=0.096)$ in the three prefectures. TB notification rates were also not significantly different between the disaster-affected areas and other areas in 2011-2013 $(P=0.115)$.

\section{DISCUSSION}

We found no TB patients had defaulted from treatment in disaster-affected areas. An increase in TB notifications was also not observed after this disaster, but TB outbreaks in shelters occurred.

Immediately after the disaster, $11.8 \%$ (45/380) of hospitals were damaged and could not receive TB patients. ${ }^{9}$ Nevertheless, the TB notification results indicated that epidemics did not occur after this disaster probably because the majority of the health systems were still well-maintained and functioning. ${ }^{10}$ The consultation meetings revealed that in the week after the disaster in Fukushima, PHC staff engaged in specific post-disaster work such as surveys of casualties and damaged medical facilities, assisting evacuation of patients from hospitals, irradiation screening for evacuees and supervision of shelters. Nevertheless from the second week onward, TB control activities were gradually resumed.

PHNs' efforts on timely resumption of TB control activities contributed to no treatment defaults. For example, in Miyagi, the PHC D building was completely immersed by water. All paper records of TB patients were lost, and all computers with patients' electronic records were damaged. Despite this situation, the PHNs conducted active patient searches to locate all 21 registered TB patients. Treatment of surviving TB patients was resumed at the end of March 2011.

Various partners, including other PHCs, medical facilities and TB patients' family members provided information on the TB patients for reporting. For example, in $\mathrm{PHC} \mathrm{G}$, one patient was missing after the tsunami. However, information of the remaining nine evacuated patients was provided by the partners and treatment continued. The successful tracking of TB patients indicated that the partners understood the necessity of reporting. Good coordination among partners also contributed to no TB treatment defaults.

The disaster-related mortality of TB patients was found to be higher than that of the general population. Although there was no evidence that TB was directly 
associated with the deaths in this disaster, co-morbidities of the TB patients might have led to inferior mobility and hindered their evacuation. Also, the mortality was found to be higher in the older age groups. ${ }^{2}$ Older people have been considered less able to make a quick evacuation. ${ }^{1}$ Special evacuation strategies should be formulated to reduce the mortality of these vulnerable groups.

To prevent TB outbreaks in shelters, information on TB prevention and diagnosis should be disseminated. In response to the first reported outbreak, JATA provided a two-page guideline for TB prevention and diagnosis at shelters in April 2011.6 Official letters were sent to the local governments to encourage its utilization.

As this study did not have comprehensive documentation for all TB patients except for treatment outcome and selected data, only some examples were reported. This may have affected the results' representativeness and accuracy.

To conclude, the results showed that post-disaster measures were effective in supporting the TB patients. TB should be included in the protocol for health care for evacuees in shelters.

\section{Conflicts of interest}

None declared.

\section{Funding}

This study was funded by the post-emergency disaster relief fund of the Japan Anti-Tuberculosis Association.

\section{Acknowledgements}

We thank staff members of Ofunato, Kamaishi and Miyako PHCs in Iwate Prefecture, of Ishinomaki, Kesennuma and Shiogama PHCs in Miyagi Prefecture and of Soso, Koriyama City and Iwaki City PHCs in Fukushima
Prefecture who participated in and provided patients' information for the cohort analysis.

\section{References}

1. Mimura $\mathrm{N}$ et al. Damage from the Great East Japan Earthquake and Tsunami - a quick report. Mitigation and Adaptation Strategies for Global Change, 2011, 16:803-818. doi:10.1007/s11027-0119297-7

2. Data on dead or missing persons by prefecture and municipality as of 11 March 2012 [In Japanese]. Tokyo, Statistics Bureau, Ministry of Internal Affairs and Communications, 2012 (http:// www.isobesatoshi.com/data/sisya-eastjapan240311.html, accessed 21 October 2015).

3. Bousai Hakusho 2011: white paper on disaster management 2011 - Executive Summary (provisional translation). Tokyo, Cabinet Office, Government of Japan, 2011 (http://www.bousai. go.jp/kaigirep/hakusho/pdf/WPDM2011_Summary.pdf, accessed 22 September 2015).

4. Anzai K et al. Fukushima Daiichi Nuclear Power Plant accident: facts, environmental contamination, possible biological effects, and countermeasures. Journal of Clinical Biochemistry and Nutrition, 2012, 50:2-8. doi:10.3164/jcbn.D-11-00021 pmid:22247595

5. Data on trend of the number of evacuees at shelters at Great East Japan Earthquake [In Japanese]. Tokyo, Cabinet Office, Government of Japan 2012 (www.bousai.go.jp/taisaku/hinanjo/ h24_kentoukai/1/pdf/8.pdf, accessed 22 September 2015).

6. Shimouchi A, Aota T, Shirai C. Large-scale disaster and tuberculosis. [In Japanese]. Nihon Kyobu Rinshou, 2012, 71:252-263.

7. Kanamori $\mathrm{H}$ et al. Tuberculosis exposure among evacuees at a shelter after earthquake, Japan, 2011. Emerging Infectious Diseases, 2013, 19:799-801. doi:10.3201/eid1905.121137 pmid:23648069

8. Yokoyama A, Abe K. Outbreak of tuberculosis in a large-size shelter after Great East Japan Earthquake [In Japanese]. Japanese Journal of Public Health, 2014, 61:527.

9. Conditions of medical facilities in disaster-affected areas as of 25 May 2011 (data provided to the Working Group of Medical Service of Social Security Council, Ministry of Health, Labour \& Welfare). Tokyo, Ministry of Health, Labor \& Welfare, 2011 (www5.cao.go.jp/npc/shiryou/goudou/pdf/3.pdf, accessed 22 September 2015).

10. Khan FA, Smith BM, Schwartzman K. Earthquake in Haiti: is the Latin American and Caribbean region's highest tuberculosis rate destined to become higher? Expert Review of Respiratory Medicine, 2010, 4:417-419. doi:10.1586/ers.10.41 pmid:20658900 